\title{
On Free-Start Collisions and Collisions for TIB3
}

\author{
Florian Mendel and Martin Schläffer \\ Institute for Applied Information Processing and Communications (IAIK) \\ Graz University of Technology, Inffeldgasse 16a, A-8010 Graz, Austria. \\ martin.schlaeffer@iaik.tugraz.at
}

\begin{abstract}
In this paper, we present free-start collisions for the TIB3 hash functions with a complexity of about $2^{32}$ compression function evaluations. By using message modification techniques the complexity can be further reduced to $2^{24}$. Furthermore, we show how to construct collisions for TIB3 slightly faster than brute force search using the fact that we can construct several (different) free-start collisions for the compression function. The complexity to construct collisions is about $2^{122.5}$ for TIB3256 and $2^{242}$ for TIB3-512 with memory requirements of $2^{53}$ and $2^{100}$ respectively. The attack shows that compression function attacks have been underestimated in the design of TIB3. Although the practicality of the proposed attacks might be debatable, they nevertheless exhibit non-random properties that are not present in the SHA-2 family.
\end{abstract}

Keywords: hash function, SHA-3 competition, TIB3, free-start collision, collision attack

\section{Introduction}

A hash function maps an input of arbitrary finite length to an output of a fixed length. An important basic security requirement for a cryptographic hash function is its collision resistance - it should be computationally infeasible to find two different inputs, which hash to the same output. Recently, the collision resistance of many commonly used hash functions has been broken or doubted. Therefore, NIST has started the SHA-3 competition [7] to find a successor of the SHA-1 and SHA-2 hash functions. The cryptanalysis of the proposed SHA-3 candidates is of high importance to find a valuable hash function which is fast but still secure within the next few decades.

Many new and interesting hash functions have been proposed and some of these algorithms have a remarkable speed on certain platforms. The SHA-3 candidate TIB3 [6] is one of the fastest submissions with a speed of about 6-8 cycles/byte for all output sizes on 64-bit platforms [3]. The main design idea behind TIB3 is to use extensive parallelism by designing a "shorter" but "wider" compression function. To strengthen this short but fast compression function and to counter differential attacks, each message block is used in two subsequent compression function calls. However, in this paper we show that it is still possible to construct collisions for the hash function TIB3 below the generic complexity. 
Table 1. Summary of results on TIB3.

\begin{tabular}{|c|c|c|c|c|}
\hline type of attack & target & hash size & complexity & memory \\
\hline \hline free-start collision & compression function & all & $2^{24}$ & - \\
collision & hash function & 256 & $2^{122.5}$ & $2^{53}$ \\
collision & hash function & 512 & $2^{242}$ & $2^{100}$ \\
\hline
\end{tabular}

Using high-probability iterative characteristics, we can construct many practical free-start collisions for the compression function of TIB3 (Sect. 3). These free-start collisions are then used for the collision attacks on both TIB3-256 (Sect. 4) and TIB3-512 (Sect. 5) with a complexity slightly below the birthday bound. The results of our work are summarized in Table 1. In the following section, we first give a short description of the hash function TIB3.

\section{Description of TIB3}

The hash function TIB3 is an iterated hash function based on the MerkleDamgård design principle [25]. The two main instances of TIB3 are called TIB3256 and TIB3-512. TIB3-256 processes message blocks of 512 bits and produces hash values of 224 or 256 bits, while TIB3-512 processes message blocks of 1024 bits and produces hash values of 384 or 512 bits. If the message length is not a multiple of the block size, an unambiguous padding method is applied. For the description of the padding method we refer to [6]. Let $m=M_{1}\left\|M_{2}\right\| \cdots \| M_{t}$ be a t-block message (after padding). Then, the hash value $h=H(m)$ is computed as follows:

$$
\begin{aligned}
H_{0} & =I V_{H}, M_{0}=I V_{M} \\
H_{i} & =f\left(H_{i-1}, M_{i} \| M_{i-1}\right) \quad \text { for } 1 \leq i \leq t \\
H_{t+1} & =f\left(H_{t}, 0\left\|H_{t}\right\| M_{t}\right)=h
\end{aligned}
$$

where $I V_{H}$ and $I V_{M}$ are predefined initial values. Note that each message block is used in two compression function calls (see Fig. 1).

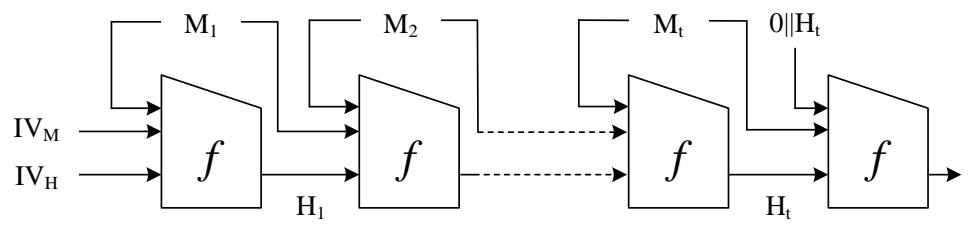

Fig. 1. The iteration mode of TIB3 uses the previous and current message block in each compression function call.

The compression function $f$ is used in Davies-Meyer mode 4] and consist of 2 parts: the key schedule and the state update transformation. The key schedule 
(or message expansion) of TIB3 takes as input the current and previous message block to compute a 4096-bit key for TIB3-256 and a 8192-bit key for TIB3-512. This key is split into 16 roundkeys $k_{j}$, where each roundkey is used in round $j$ of the state update transformation. For a detailed description of the key schedule function we refer to [6], since we do not need it in our analysis. In the following, we describe the state update transformation for TIB3-256 and TIB3-512 in more detail.

\subsection{State Update Transformation for TIB3-256}

The state update transformation of TIB3-256 starts from a (fixed) initial value $I V_{H}$ of four 64-bit words and updates them in 16 rounds each. In each round one 256-bit roundkey $k_{j}$ is used to update the four state variables $A, C, E$ and $G$ as follows:

$$
\begin{aligned}
G & =G \oplus C \\
(A, C, E, G) & =(A, C, E, G) \oplus k_{j} \\
(A, C, E) & =\operatorname{Sbox}(A, C, E) \\
G & =\operatorname{PHTX}(G) \\
C & =\operatorname{PHTX}(C) \\
A & =A \boxplus^{32} G \\
G & =E \boxplus^{32} G \\
(A, C, E, G) & =(C, E, G, A),
\end{aligned}
$$

where $S b o x$ is a 3-bit S-box, PHTX is a bit-mixing function and $\boxplus^{32}$ denotes two 32-bit modular additions in parallel. One round of the TIB3-256 compression function is shown in Fig. 2 For the definition of the S-boxes we refer to [6]. The function $O=\operatorname{PHTX}(I)$ is defined as follows:

$$
\begin{aligned}
& T=I+(I \ll 32)+(I \ll 47) \\
& O=T \oplus(T \gg 32) \oplus(T \gg 43)
\end{aligned}
$$

After the last round of the state update transformation, the chaining values $A_{0}, C_{0}, E_{0}, G_{0}$ are XORed with the output values of the last round $A_{16}, C_{16}$, $E_{16}, F_{16}$ (feed-forward), resulting in the final value of one compression function $f$. For a detailed description of the hash function we refer to [6].

\subsection{State Update Transformation for TIB3-512}

In TIB3-512, two instances of the TIB-256 compression function are computed in parallel. The two parallel instances are mixed by two PHTXD functions with inputs $C, D$ and $G, H$. A short description of the state update of TIB3-512 is given below, for more details we refer to $[\underline{6}$. 


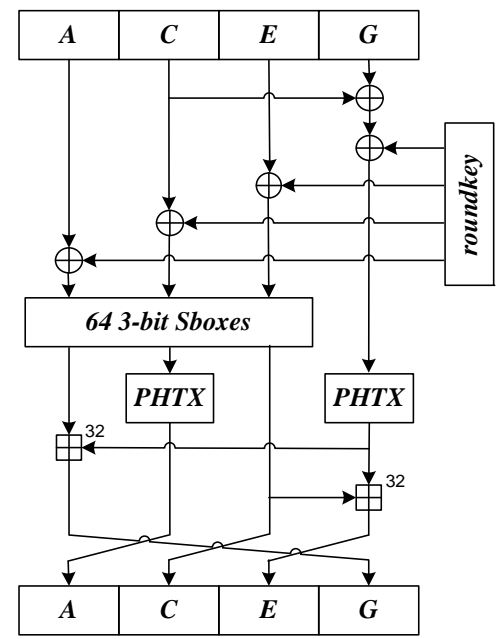

Fig. 2. One round of the TIB3-256 compression function.

The state update transformation of TIB3-512 updates eight 64-bit words $A$, $B, C, D, E, F, G$ and $H$ in 16 rounds. One round of TIB3-512 is shown in Fig. 3 and defined as follows:

$$
\begin{aligned}
G & =G \oplus C \\
H & =H \oplus D \\
(A, B, C, D, E, F, G, H) & =(A, B, C, D, E, F, G, H) \oplus k_{j} \\
(A, C, E) & =\operatorname{Sbox}(A, C, E) \\
(B, D, F) & =\operatorname{Sbox}(B, D, F) \\
(G, H) & =\operatorname{PHTX} D(G, H) \\
(C, D) & =P H T X D(C, D) \\
A & =A \boxplus G \\
B & =B \boxplus H \\
G & =E \boxplus G \\
H & =F \boxplus H \\
(A, B, C, D, E, F, G, H) & =(C, D, E, F, G, H, A, B),
\end{aligned}
$$

where $k_{j}$ is the current 512-bit roundkey, $S b o x$ is the same 3-bit S-box as in TIB3-256 and $\boxplus$ denotes a 64 -bit modular addition. The function $(O, P)=$ $\operatorname{PHTXD}(I, J)$ is a "double" version of PHTX and is defined as follows:

$$
\begin{aligned}
& P=I \oplus J \\
& P=P H T X(P) \\
& O=I \oplus P \\
& O=P H T X(O)
\end{aligned}
$$




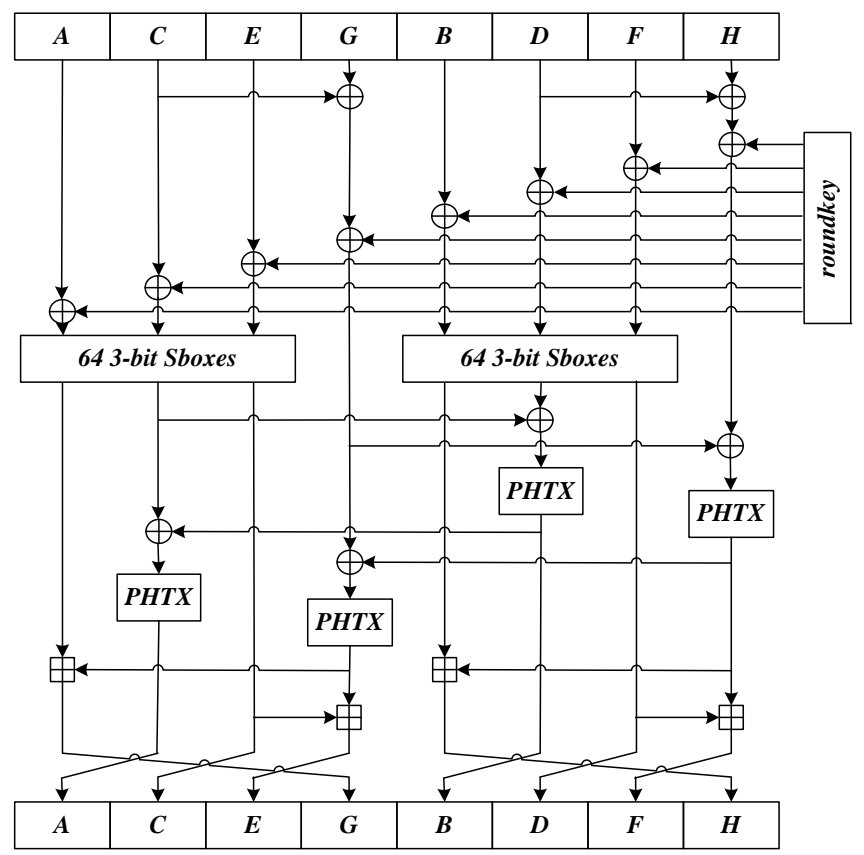

Fig. 3. One round of the TIB3-512 compression function.

\section{Free-Start Collisions for TIB3-256}

In this section, we present a free-start collision attack on the compression function of TIB3-256 with a complexity of about $2^{24}$ compression function evaluations. Note that we use only differences in the chaining inputs and no differences in the message inputs are allowed. This is similar to the attack of den Boer and Bosselaers on MD5 [1]. However, in the case of TIB3 the complexity of the attack is much better due to its short compression function.

The attack is based on the fact that we can construct several 1-round iterative characteristics for the compression function of TIB3-256 with a probability between $2^{-2}$ and $2^{-4}$, depending on the bit position of the differences. The 1-round characteristic is shown below:

$$
(-, \Delta[i], \Delta[i], \Delta[i]) \rightarrow(-, \Delta[i], \Delta[i], \Delta[i])
$$

where $\Delta[i]$ denotes a difference at bit position $i$. By subsequently using this 1-round characteristic 16 times, we will get a free-start collision for the whole 16-round compression function of TIB3-256. Note that the differences of the last round in $C_{16}, E_{16}$ and $G_{16}$ will be canceled due to the feed-forward, i.e. $A_{0} \oplus A_{16}$, $C_{0} \oplus C_{16}, E_{0} \oplus E_{16}$, and $G_{0} \oplus G_{16}$. 
Table 2. Differential distribution table for the 3-bit S-box of TIB3 (cf. 6, page 15]) with input difference $S_{i}=(A, C, E)$ and corresponding output difference $S_{o}$. Probabilities are given in base 2 logarithms.

\begin{tabular}{c|ccccccc}
$S_{i} \backslash S_{o}$ & 001 & 010 & 011 & 100 & 101 & 110 & 111 \\
\hline 001 & & -2 & -2 & & & -2 & -2 \\
010 & -2 & & -2 & & -2 & & -2 \\
011 & -2 & -2 & & & -2 & -2 & \\
100 & & & & -2 & -2 & -2 & -2 \\
101 & & -2 & -2 & -2 & -2 & & \\
110 & -2 & & -2 & -2 & & -2 & \\
111 & -2 & -2 & & -2 & & & -2
\end{tabular}

\subsection{On the Probability of the Characteristic}

Now, lets take a closer look at the probability of the characteristic for each round $j$ which is shown in Fig. 4. Note that the xor of the roundkey in each round never changes the difference. The probabilities for all input/output differences of the 3 -bit S-box of TIB3 are shown in Table 2 .

- We start with the differences $\Delta[i]$ in $C, E$ and $G$. After the first xor operation, the difference in $G$ is canceled. In order to guarantee that the characteristic holds, we need that the differences $\Delta[i]$ in $C$ and $E$ at the input of the S-box propagate to the differences $\Delta[i]$ in $A$ and $E$ after the S-box. This holds with a probability of $2^{-2}$, see Table 2 .

- Note that there are no differences in the PHTX functions.

- In the case of $i=\{32,64\}$, no carry occurs in the four 32-bit modular additions and the differences $\Delta[i]$ in $A$ and $E$ propagate to $\Delta[i]$ in $G, C$ and $E$ with a probability of 1 . In the case of $i \neq\{32,64\}$ no carry occurs in the two additions with a probability of $2^{-2}$.

- Hence, the resulting difference $\Delta[i]$ in $C, E$ and $G$ after one round is the same as in the input to this round.

The characteristic holds for one round with a probability of $2^{-2}$ for $i=\{32,64\}$ and $2^{-4}$ for $i \neq\{32,64\}$ and we get a characteristic for all 16 rounds with a probability of $2^{-32}$ and $2^{-64}$, respectively. Thus, we can construct a free-start collision for the compression function of TIB3-256 with a complexity of about $2^{32}$ for $i=\{32,64\}$ and $2^{64}$ for $i \neq\{32,64\}$ instead of $2^{128}$ as expected for a compression function with 256 bits. An example for a free-start collision for TIB3-256 with $i=64$ is given in Table 3 .

\subsection{Improving the Attack Complexity}

The complexity of the free-start collision attack can be significantly improved by using message modification techniques. Message modification was introduced by Wang et al. in the cryptanalysis of MD5 and SHA-1 [1011]. The idea of message 


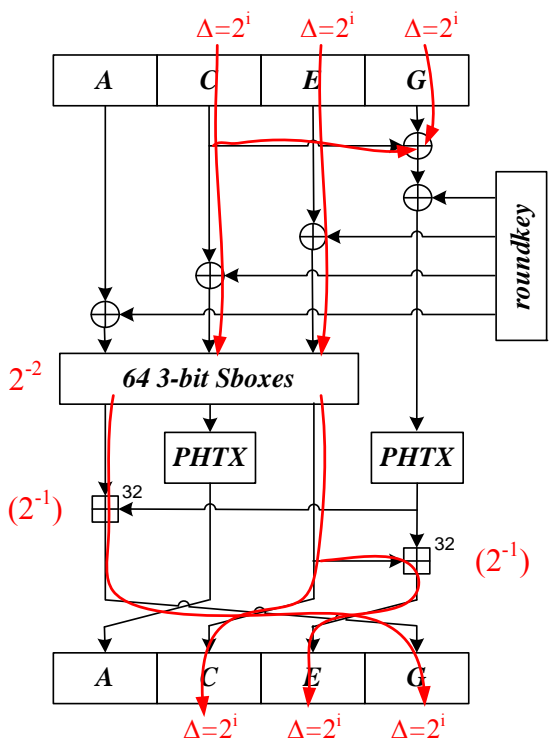

Fig. 4. The 1-round iterative differential characteristic for TIB3-256 which completely avoids the PHTX functions. We get a differential probability of $2^{-2}$ for the S-box, and $2^{-1}$ for the modular addition if $i \neq\{32,64\}$.

modification is to use the degrees of freedom one has in the choice of the message words to fulfill conditions on the chaining variables.

In the case of TIB3-256 we can at least use the current 1024 bit message block for message modification. Using these 1024 degrees of freedom, the 16 conditions in the first 4 rounds can be fulfilled using basic message modification. In other words, we do not care about the probability of the characteristic in this part, since a message following the characteristic in the first 4 rounds can be found deterministically. Hence, the complexity of the attack can be reduced to $2^{24}$ for $i=\{32,64\}$ and to $2^{48}$ for $i \neq\{32,64\}$. We expect that the complexity can be further improved by using more sophisticated message modification techniques.

\section{Collision Attack for TIB3-256}

In this section, we show how the free-start collision attack on the compression function can be extended to a collision attack on the hash function. Even though the complexity of the attack is only slightly faster than a generic birthday attack, it exhibits some non-random properties that are not present in SHA-256. The attack uses the fact, that we can find several high-probability free-start collision producing characteristics for the compression function of TIB3-256. 
Table 3. A free-start collision for TIB3-256 with differences at bit position 64 .

\begin{tabular}{|c|c|c|}
\hline$H_{1}^{\prime}$ & $H_{1}^{\prime \prime}$ & $\Delta H_{1}$ \\
\hline 0000000000000000 & 0000000000000000 & 0000000000000000 \\
\hline $0000000 \quad 00000000$ & 8000000000000000 & 8000000000 \\
\hline 0000000000000000 & 8000000000000000 & 8000000000000000 \\
\hline 000000000000000 & 8000000000000000 & 8000000000000000 \\
\hline$M_{1}$ & $M_{2}$ & $\Delta M_{1}, \Delta M_{2}$ \\
\hline OBDD5C0 $451 \mathrm{CE} 787$ & E75BFF16 FACB4B84 & 00000000000 \\
\hline 6BB03ABE 814 & 6D6A0C85 52A & 000000 \\
\hline F45283B2 4019E54C & AECE5E32 A5F07508 & 0000000000000000 \\
\hline 68D47A8C EC6 & A64F3E2B E51 & 00000000000 \\
\hline 20AC1B8D 5C4F42F0 & E5079CCA 5CC28EBE & 00000000000 \\
\hline B239522C 8BF26045 & 1E7E2827 4E8C6B37 & 0000000000000000 \\
\hline E0EC45C2 3ACE0DE7 & $808 \mathrm{C} 0 \mathrm{~A} 2 \mathrm{~F}$ B5E1F9AA & 0000000000000000 \\
\hline 2FB7DEBD 84DDCF 10 & 3BBF29A5 FAB148DF & 0000000000000000 \\
\hline$H_{2}^{\prime}$ & $H_{2}^{\prime \prime}$ & $\Delta H_{2}$ \\
\hline 55F5547C 6AA5CC12 & $55 \mathrm{~F} 5547 \mathrm{C}$ 6AA5CC12 & 00000000000 \\
\hline 40831045 5CC5F776 & 40831045 5CC5F776 & 00000000000 \\
\hline 43E53C0C 4C64F862 & 43E53C0C 4C64F862 & 0000000000000000 \\
\hline DD750B01 DA7AD37F & DD750B01 DA7AD37F & 0000000000000000 \\
\hline
\end{tabular}

\subsection{Increasing the Number of Free-Start Collisions}

In the previous section, we have constructed 64 different free-start collisions for $i=1, \ldots, 64$. To increase the number of characteristics, we can fit two high probability characteristics with bit position $i \neq j$ into the compression function:

$$
(-, \Delta[i, j], \Delta[i, j], \Delta[i, j]) \rightarrow(-, \Delta[i, j], \Delta[i, j], \Delta[i, j])
$$

In the case of $i, j \neq\{32,64\}$, we get a total probability of $2^{-128}$ which can be reduced to $2^{-96}$ by message modification. Note that we can further increase the number of characteristics by allowing carries at the beginning (first rounds) and end (last rounds). Hence, we can construct at least $\left(\begin{array}{c}64 \\ 2\end{array}\right) \sim 2^{11}$ different free-start collision.

\subsection{From Free-Start Collisions to Collisions}

In this section, we show how to use $2^{x}$ free-start collisions of the compression function to find collisions for the full hash function with a complexity of $2^{\frac{n-x}{2}}$. In the case of TIB3-256 we have constructed $2^{11}$ free-start collisions characteristics. Hence, the collision attack on TIB3-256 has a complexity of about $2^{122.5}$ compression function calls.

The collision attack uses 3 message blocks $M_{1}, M_{2}$ and $M_{3}$ (see Fig. 5). The main idea of the attack is to find two different first message blocks $M_{1}^{\prime}$ and $M_{1}^{\prime \prime}$ which result in one of the $2^{11}$ differences of the free-start collision in $H_{2}$. Then, the according free-start collision is used to get a collision in $H_{3}$ after the second 


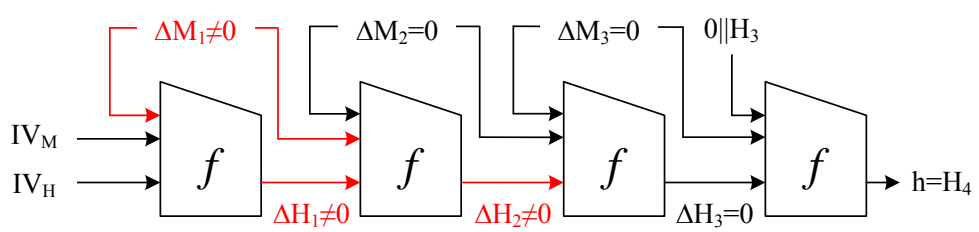

Fig. 5. In the collision attack on TIB3, three message blocks are used with differences only in $M_{1}$. The resulting near-collision in $H_{2}$ is transformed into a collision in $H_{3}$. The last message block $M_{3}$ is used for message modification.

compression function call. Note that we need a third message block $M_{3}$ for the message modification of the free-start collision:

$$
\begin{aligned}
& H_{1}=f\left(I V_{H}, M_{1} \| I V_{M}\right) \\
& H_{2}=f\left(H_{1}, M_{2} \| M_{1}\right) \\
& H_{3}=f\left(H_{2}, M_{3} \| M_{2}\right) \\
& H_{4}=f\left(H_{3}, 0\left\|H_{3}\right\| M_{3}\right)=h
\end{aligned}
$$

The collision attack on TIB3-256 can then be summarized as follows:

1. Choose an arbitrary value for the message block $M_{2}$.

2. Use a birthday attack to find a $\Delta H_{2}$ (near-collision) which matches one of the $2^{11}$ free-start collision producing characteristics. Note that $M_{2}$ is fixed in the attack and only $M_{1}$ can be modified. This is important, since we do not allow any differences in $M_{2}$. The birthday phase has a complexity of about $2^{\frac{256-11}{2}}=2^{122.5}$ compression function evaluations and can be implemented as follows:

(a) Choose $2^{122.5}$ different random message blocks $M_{1}$ and store the messages and resulting chaining values $H_{2}$ in a set $S$. Then, we need to find those two messages $M_{1}^{\prime}, M_{1}^{\prime \prime}$ with $M_{1}^{\prime} \oplus M_{1}^{\prime \prime}=\Delta M_{1}$, such that $H_{2}^{\prime} \oplus H_{2}^{\prime \prime}=\Delta H_{2}$ belongs to the set of $2^{11}$ free-start collision producing characteristics:

(b) From the set $S$ find all pairs $\left(M_{1}^{\prime}, M_{1}^{\prime \prime}\right)$ with $H_{2}^{\prime}=A^{\prime}\left\|C^{\prime}\right\| E^{\prime} \| G^{\prime}$ and $H_{2}^{\prime \prime}=A^{\prime \prime}\left\|C^{\prime \prime}\right\| E^{\prime \prime} \| G^{\prime \prime}$ such that $A^{\prime}=A^{\prime \prime},\left(C^{\prime} \oplus E^{\prime}\right)=\left(C^{\prime \prime} \oplus E^{\prime \prime}\right)$ and $\left(C^{\prime} \oplus G^{\prime}\right)=\left(C^{\prime \prime} \oplus G^{\prime \prime}\right)$. Note that this can be done with a standard birthday attack (using a hash table) and we get $2^{122.5} \times 2^{122.5} \times 2^{-192}=$ $2^{53}$ pairs.

(c) For each pair, compute $C^{\prime} \oplus C^{\prime \prime}$ and check if the Hamming weight $H W\left(C^{\prime} \oplus C^{\prime \prime}\right) \leq 2$. In other words, check if $C^{\prime} \oplus C^{\prime \prime}$ (and hence, $H_{2}^{\prime} \oplus H_{2}^{\prime \prime}$ ) belongs to the set of $2^{11}$ free-start collision producing characteristics. This step of the attack has a complexity of about $2^{64-11}=2^{53}$ XOR operations which is negligible compared to $2^{122.5}$ compression function evaluations.

3. Finally, we use the according free-start collision producing characteristic to turn the near-collision of $\Delta H_{2}$ into a collision in $H_{3}$ by using the message 
blocks $M_{2}$ and $M_{3}$. Note that there are no differences in these two message blocks, which is needed for the free-start collision producing characteristic to work ( $c f$. Section 3). Note that $M_{3}$ can still be chosen freely in the attack and hence, used for message modification. This step of the attack has a complexity of about $2^{96}$ compression function evaluations.

Alltogether, we can construct collisions in TIB3-256 with a complexity of about $2^{122.5}$ compression function evaluations and similar memory requirements. The complexity of this attack can be improved as soon as more than $2^{11}$ free-start collision characteristics have been constructed. One possibility to increase the number of characteristics is by allowing carries at the beginning (first rounds) and end (last rounds) of the compression function. Furthermore, the memory requirements of the attack can be significantly reduced. By using distinguished points 8 89 the first part of the birthday attack, i.e. 2.(a)-2.(b) can be implemented with memory requirements of $2^{53}$ instead of $2^{122.5}$.

\section{Collision Attack for TIB3-512}

The collision attack on TIB-256 can be extended to the hash function TIB3-512 as well. Since TIB3-512 uses two instances of TIB3-256 in parallel, we can reuse the free-start collision producing characteristic of TIB3-256 (Sect. 3).

\subsection{Free-Start Collisions for TIB3-512}

Since we do not have differences at the input of any PHTXD (mixing) function, the same free-start collisions of TIB3-256 can indeed be used without modification for TIB3-512. Hence, we can construct two different and independent 1-round characteristic for TIB-512 with differences in $C, E, G$ :

$$
(-,-, \Delta[i],-, \Delta[i],-, \Delta[i],-) \rightarrow(-,-, \Delta[i],-, \Delta[i],-, \Delta[i],-)
$$

and/or with differences in $D, F, H$ :

$$
(-,-,-, \Delta[i],-, \Delta[i],-, \Delta[i]) \rightarrow(-,-,-, \Delta[i],-, \Delta[i],-, \Delta[i])
$$

where $\Delta[i]$ denotes a difference at bit position $i$. The probability is $2^{-4}$ for $i \neq 64$ and $2^{-2}$ for $i=64$ since one 64 -bit addition is used instead of two 32bit additions. TIB-512 has 16 rounds as well and we can construct a free-start collision for the compression function of TIB3-512 with a complexity of $2^{32}$ for $i=64$ and $2^{64}$ for $i \neq 64$ again. Using basic message modification we can reduce the complexity to $2^{24}$ and $2^{48}$ respectively. An example for a free-start collision with a difference in bit position 64 is given in Table 4

\subsection{From Free-Start Collisions to Collisions}

In the case of TIB3-512, the generic complexity for a collision attack is $2^{256}$. Therefore, we can easily fit up to 5 high probability characteristics next to each 
Table 4. A free-start collision for TIB3-512 with differences at bit position 64 .

\begin{tabular}{|c|c|c|}
\hline$H_{1}^{\prime}$ & $H_{1}^{\prime \prime}$ & $\Delta H_{1}$ \\
\hline 0000000000000000 & 0000000000000000 & 0000000000000000 \\
\hline 000000000000000 & 0000000000000 & 0000000000 \\
\hline 0000000000000000 & 0000000000000000 & $00000000 \quad 00000000$ \\
\hline 0000000000000000 & 8000000000000000 & 8000000000000000 \\
\hline 0000000000000000 & 00000000000 & 00000000000 \\
\hline 0000000000000000 & 8000000000000000 & 80000000000 \\
\hline 0000000000000000 & 0000000000000 & 0000000000000000 \\
\hline 0000000000000000 & 8000000000000 & 80000000000 \\
\hline$M_{1}$ & $M_{2}$ & $\Delta M_{1}, \Delta M_{2}$ \\
\hline 246B6D96 2C9C & $240 \mathrm{E} 562 \mathrm{C} 5 \mathrm{C}$ & 0000000 \\
\hline 6139BD35 C099E9CC & 31СОАЗВО ВЗС & 00000 \\
\hline 5533B6BF D6B80FB1 & 94E6BEBD 91BC6264 & 0000000000000000 \\
\hline 099868E2 8C9A5821 & BB665DC4 B5C & 00000 \\
\hline 08ED963E A808F1E6 & 7AEFABF8 3DF & 000000 \\
\hline 1658D8E1 94925F32 & A4D3961F 2C8BFCF8 & 000000 \\
\hline AF7DE86F $4013 C A D 4$ & 626DED61 3B3B & 000000 \\
\hline 24573C4C 867D59A2 & 873613B2 C1F4B14A & 0000000000000000 \\
\hline$H_{2}^{\prime}$ & $H_{2}^{\prime \prime}$ & $\Delta H_{2}$ \\
\hline $8011137 \mathrm{D} 30451 \mathrm{AAO}$ & & 0000000000000000 \\
\hline 5791600A B98C1C4A & $5791600 \mathrm{~A} \quad \mathrm{~B} 9$ & 00000000000 \\
\hline 60570740 31EEA496 & 60570740 31EEA496 & 0000000000000000 \\
\hline 31FB13D0 8A58960D & 31FB13D0 8A589 & 000000 \\
\hline 15C9B361 99054AB7 & 15С9B361 99054AB7 & 0000000000000000 \\
\hline B6312CAB 57CF73AE & B6312CAB 57CF73AE & 0000000000000000 \\
\hline C7055809 B6B3BB6A & С7055809 В6В3ВВ6А & 0000000000000000 \\
\hline 422F8F0B 9DCCC9A4 & 422F8F0B 9DCCC9A4 & 0000000000000000 \\
\hline
\end{tabular}

other (complexity $2^{5 \cdot 48}=2^{240}$ ). Hence, we can construct at least $\left(\begin{array}{c}128 \\ 5\end{array}\right) \sim 2^{28}$ different free-start collision producing characteristics for TIB3-512. The resulting collision attack has a complexity of about $2^{\frac{512-28}{2}}=2^{242}$ and memory requirements of about $2^{128-28}=2^{100}$ using distinguished points.

\section{Conclusion}

In this paper, we have presented free-start collisions for TIB3 with a complexity of about $2^{32}$ compression function evaluations. By using message modification techniques the complexity can be reduced to $2^{24}$. Furthermore, we can construct at least $2^{11}$ free-start collision producing characteristics for TIB3-256 and $2^{28}$ for TIB3-512. We show how to use these free-start collisions to construct collisions for TIB3 slightly faster than brute force search, and get a complexity of about $2^{122.5}$ compression function calls for TIB3-256 and $2^{242}$ for TIB3-512 with memory requirements of $2^{53}$ and $2^{100}$, respectively.

TIB3 is one of the fastest submissions due to its parallelism but short compression function. In the design of TIB3, compression function attacks have been 
underestimated. In this paper, we have shown how to find high-probability freestart collisions and turn them into an attack on the hash function. Although the practicality of the proposed attacks might be debatable, they nevertheless exhibit non-random properties that are not present in the SHA-2 family. Since there is still room for improvements, this analysis can be a starting point for future attacks on TIB3.

\section{Acknowledgements}

We thank the designers of TIB3 for useful discussions and pointing out a small error in a previous version of this paper. The work in this paper has been supported by the European Commission under contract ICT-2007-216646 (ECRYPT II).

\section{References}

1. den Boer, B., Bosselaers, A.: Collisions for the Compression Function of MD5. In: Helleseth, T. (ed.) EUROCRYPT. LNCS, vol. 765, pp. 293-304. Springer (1993)

2. Damgård, I.: A Design Principle for Hash Functions. In: Brassard, G. (ed.) CRYPTO. LNCS, vol. 435, pp. 416-427. Springer (1989)

3. Fleischmann, E., Forler, C., Gorski, M.: Classification of the SHA-3 Candidates. Cryptology ePrint Archive, Report 2008/511 (2008), http://eprint.iacr.org

4. Matyas, S.M., Meyer, C.H., Oseas, J.: Generating strong one-way functions with crypographic algorithm. IBM Technical Disclosure Bulletin 27(10A), 5658-5659 (1985)

5. Merkle, R.C.: One Way Hash Functions and DES. In: Brassard, G. (ed.) CRYPTO. LNCS, vol. 435, pp. 428-446. Springer (1989)

6. Montes, M., Penazzi, D.: The TIB3 Hash. Submission to NIST (2008)

7. NIST: Announcing Request for Candidate Algorithm Nominations for a New Cryptographic Hash Algorithm (SHA-3) Family. Federal Register Notice (November 2007), available online at: http://csrc.nist.gov

8. van Oorschot, P.C., Wiener, M.J.: Parallel Collision Search with Cryptanalytic Applications. J. Cryptology 12(1), 1-28 (1999)

9. Quisquater, J.J., Delescaille, J.P.: How Easy is Collision Search. New Results and Applications to DES. In: Brassard, G. (ed.) CRYPTO. LNCS, vol. 435, pp. 408413. Springer (1989)

10. Wang, X., Yin, Y.L., Yu, H.: Finding Collisions in the Full SHA-1. In: Shoup, V. (ed.) CRYPTO. LNCS, vol. 3621, pp. 17-36. Springer (2005)

11. Wang, X., Yu, H.: How to Break MD5 and Other Hash Functions. In: Cramer, R. (ed.) EUROCRYPT. LNCS, vol. 3494, pp. 19-35. Springer (2005) 